\title{
Guidance of an Autonomous Agent for Coverage Applications using Range Only Measurement
}

\author{
Twinkle Tripathy* and Arpita Sinha ${ }^{\dagger}$ \\ Systems \& Control Engineering, IIT Bombay, Mumbai, Maharashtra, 4000\%6, India
}

\begin{abstract}
This paper addresses the problem of guiding an autonomous agent to cover a region surrounding a target. It is assumed that only range information is available to the agent. The target point is considered to be stationary. The region of interest can be changed by changing the initial conditions or the system parameters. In this work, we analyse the effect of the initial conditions only. We propose a simple control law which can be easily implemented. The analytical results obtained here are validated through simulation. The autonomous agent could be a ground or an aerial vehicle depending on the application. For surveillance applications, the target can be a landmark, where the agent can monitor the region surrounding the landmark. Other applications can be sprinkling fertilizers, watering a farm, etc. where a pre-defined area needs to be covered.
\end{abstract}

\section{Introduction}

$\mathrm{T}$ HE coverage problem refers to the problem of guiding an autonomous agent to cover an unknown or predefined area. Several techniques have been proposed to achieve coverage, which vary depending on the application. There are several algorithms which are used to cover unknown areas. For example, a lot of work has been done on map based coverage techniques (see [1], [2], [3], [4] and references therein) which work by dividing the area into cells and then assigning values to the cells based on the presence of obstacles or free space. Wong et al. ${ }^{1}$ used topological maps to solve the coverage problem and their results are verified by simulation tests which show that over $99 \%$ of the surface is covered. The topological maps, represent the environment as graphs where landmarks are nodes, and edges represent the connectivity between the landmarks. The algorithm achieves coverage with single mobile agent. Zelinsky et al. ${ }^{2}$ also use maps for coverage but with a different approach. The solution ensures complete coverage by the use of a distance transform path planning methodology. The solution was simulated and implemented on an autonomous robot called Yamabico to give satisfactory performance. Stachniss et al. ${ }^{3}$ introduced the concept of coverage maps where each cell of a given grid corresponds to the amount of a cell which is covered by an obstacle; the coverage maps are improvement of occupancy grids which are based on the assumption that each cell is either occupied or free. The model presented in the paper allows updation of the coverage maps upon input obtained from sensors. Rutishauser et al. ${ }^{4}$ solve the problem of collaborative coverage using a swarm of networked miniature robots again by the use of grid based methods. For a multi-agent system, the problem of coverage, addressed by Batavia et al., ${ }^{5}$ has been solved with high accuracy for a semi- structured environment. The approach has the advantage of an operator driving the outline of a desired coverage area as input to a coverage generation algorithm. Acar et al. ${ }^{6}$ have solved the coverage problem by the decomposition of the environment, by the use of voronoi diagrams.

Surveillance based systems, discussed in [7], [8] and [9], use wireless sensor networks to solve the coverage problem. The sensor nodes are capable of behaving autonomously. In [7] and [9], the coverage achieved is average, but more emphasis is laid on the placement of sensors for effective coverage; Dhillon et al. ${ }^{7}$ have based their work on fixed sensor nodes. Lai et al. ${ }^{8}$ discuss surveillance systems based on wireless sensor networks. In [8], the deployed sensors are divided into disjoint subsets of sensors, or sensor covers, such that each sensor cover can cover all targets and work in turns. A surveillance system based on wireless

\footnotetext{
*Research Scholar, Systems \& Control Engineering, IIT Bombay and Student Member, AIAA.

${ }^{\dagger}$ Assistant Professor, Systems \& Control Engineering, IIT Bombay
} 


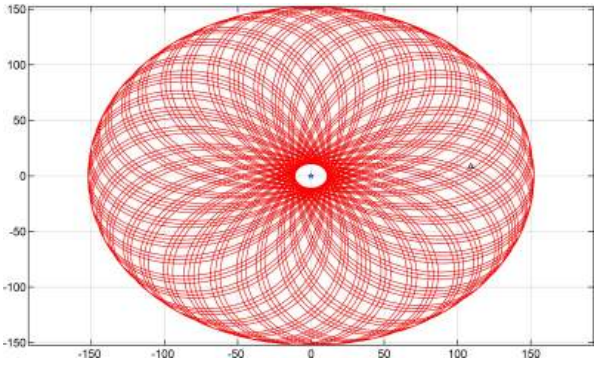

Figure 1: Coverage obtained by an Autonomous Agent

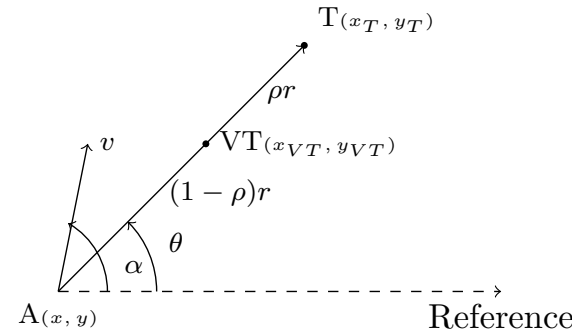

Figure 2: Engagement geometry between the agent and the target point

sensor networks has been discussed by Yan et al. ${ }^{10}$ The system provides a differentiated surveillance system which deploys sensors nodes in appropriate areas depending on the degree of surveillance required. But, the wireless sensor networks suffer from the problem of energy requirements which increase with the number of sensor nodes used in the network.

The problem discussed in this paper is formulated as follows. The autonomous agent is modeled as an unicycle. We assume, the region to be covered, is circular or annular around the target as shown in Fig. 3. The agent uses only distance information to cover the annular region. The annular region is specified by $R_{\min }$ and $R_{\max }$. It is shown that $R_{\min }$ and $R_{\max }$ depend on the initial conditions. Using this, we have derived:

- The $R_{\min }$ and $R_{\max }$, given any initial conditions.

- The initial conditions necessary to achieve a given $R_{\min }$ or $R_{\max }$.

This paper is organized as follows. In Section II, we define the problem and analyze it in Section III. Section IV elaborates the analysis further for a particular situation. Simulations results are presented in Section V and Section VI concludes the paper and discusses the areas on which further work can be done in future.

\section{Problem Statement}

The paper addresses the coverage problem as shown in Fig. 3. Let the target be at $\left(x_{T}, y_{T}\right)$ and is stationary at any instant $t>0$, position of agent is given by $(x(t), y(t))$, as shown in Fig. 2. The kinematics of agent is as shown below:

$$
\begin{array}{r}
\dot{x}(t)=v \cos \alpha(t) \\
\dot{y}(t)=v \sin \alpha(t) \\
\dot{\alpha}(t)=u(t)
\end{array}
$$

where $v$ is the linear velocity, which is a constant for our case, $\alpha(t)$ is the heading angle and $u(t)$ is the control input. In Fig. 2, $r(t)$ is the line-of-sight(LOS) distance and $\theta(t)$ is the LOS angle. Since only range measurement is feasible, agent knows only instantaneous $r(t)$ and not $\theta(t)$. Let $V T$ denote a virtual point, called the virtual target which is at a distance $\rho r(t)$ from the target along the LOS, where $\rho \in(0,1)$. In the control law, the lateral acceleration of the autonomous agent is made proportional to the distance between the virtual target and the agent, given by $\left(\left(x_{V T}-x(t)\right)^{2}+\left(y_{V T}-y(t)\right)^{2}\right)^{1 / 2}=(1-\rho) r(t)$. So, the algorithm is defined in terms of $a_{M}(t)$ as:

$$
\begin{aligned}
& a_{M}(t)=h(1-\rho) r(t) \\
& \dot{\alpha}(t)=\frac{a_{M}(t)}{v}=k r(t)
\end{aligned}
$$

where $h$ is the constant of proportionality between $a_{M}$ and the distance, $(1-\rho) r$, and $k=\frac{h(1-\rho)}{v}$. The parameter $h$ is a system gain. The concept of the virtual target increases the flexibility of the algorithm 


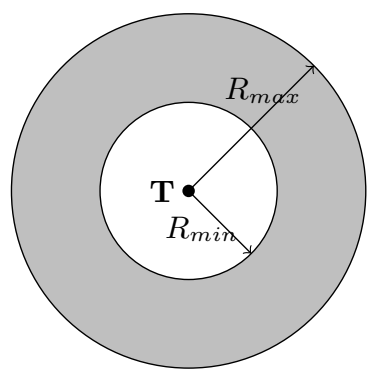

Figure 3: Region to be covered

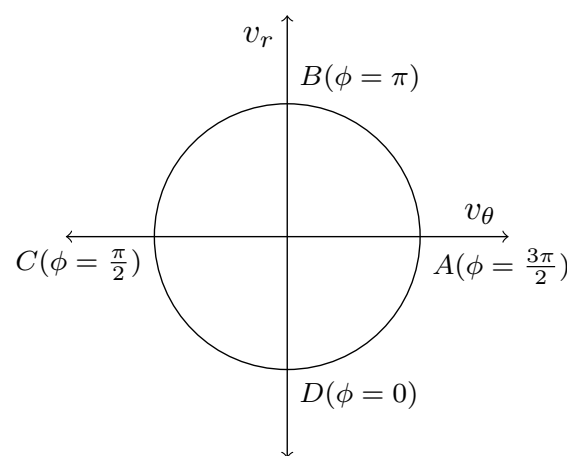

Figure 4: $\left(v_{\theta}, v_{r}\right)$ plot

as we get another parameter $\rho$ to be varied as needed. The algorithm generates various coverage patterns surrounding the stationary target. One of the patterns generated is shown in Fig. 1. In the next section, the control algorithm is analysed for area coverage applications.

\section{Analysis}

Consider the Fig. 2, the LOS between the agent and the target can be charaterised as follows:

$$
\begin{gathered}
v_{r}=\dot{r}=-v \cos (\alpha-\theta) \\
v_{\theta}=r \dot{\theta}=-v \sin (\alpha-\theta)
\end{gathered}
$$

Let, $\phi=\alpha-\theta$. From eqns. (5)-(7), we get,

$$
\dot{\phi}=\dot{\alpha}-\dot{\theta}=k r+\frac{v \sin \phi}{r}
$$

From eqns. (6) and (8),

$$
\left(k r^{2}-v_{\theta}\right) d r+v r \cos (\phi) d \phi=0
$$

Solving the integration, we establish a relationship between $r$ and $\phi$ as,

$$
v r \sin \phi+\frac{k r^{3}}{3}=v r_{0} \sin \phi_{0}+\frac{k r_{0}^{3}}{3}
$$

In the above equation, $r_{0}$ and $\phi_{0}$ are the initial conditions. We define a variable $K$ which contains all the terms corresponding to the initial conditions, such that,

$$
K=\left(\frac{3 v r_{0} \sin \left(\phi_{0}\right)}{k}+r_{0}^{3}\right)
$$

Hence, Eqn. (10) can be re-written as:

$$
r^{3}+\frac{3 v r \sin \phi}{k}-K=0
$$

By setting Egn. (6) to zero, we observe that $r$ can take its maximum and minimum values that is $R_{m i n}$ and $R_{\max }$ when $\phi$ equals either $\frac{3 \pi}{2}$ or $\frac{\pi}{2}$. Next we look into how the kinematics varies in the $\left(v_{\theta}, v_{r}\right)$ plane. Upon squaring and adding Eqn. (6) and Eqn. (7), we find that the instantaneous $v_{\theta}$ and $v_{r}$ lie on a circle in the $\left(v_{\theta}, v_{r}\right)$ plane as shown in Fig. 4. By the use of Eqns. (6) and (7), we can calculate the value of $\phi$ corresponding to each set of instantaneous $v_{r}$ and $v_{\theta}$. Point $A$ corresponds to $\left(v_{\theta}, v_{r}\right)=(v, 0)$, thus, $\phi=\frac{3 \pi}{2}$. Similarly, we get that at $B, \phi=\pi$, at $C, \phi=\frac{\pi}{2}$ and at $D, \phi=0$. Hence, the clockwise movement about the circle shown in Fig. 4 gives the direction of increasing values $\phi$. Combining Eqns. (8) and (12), we get,

$$
\frac{d \phi}{d t}=\frac{k\left(K+2 r^{3}\right)}{3 r^{2}}
$$




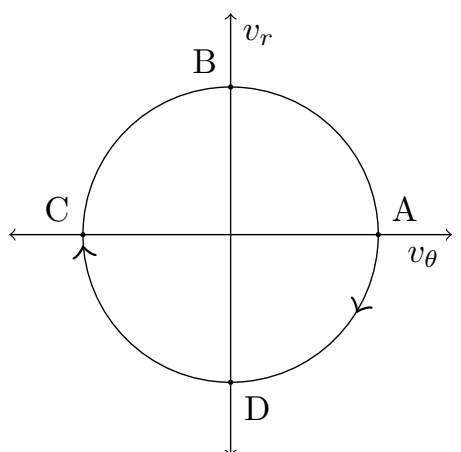

Figure 5: Engagement trajectory 1 in $\left(v_{\theta}, v_{r}\right)$ space

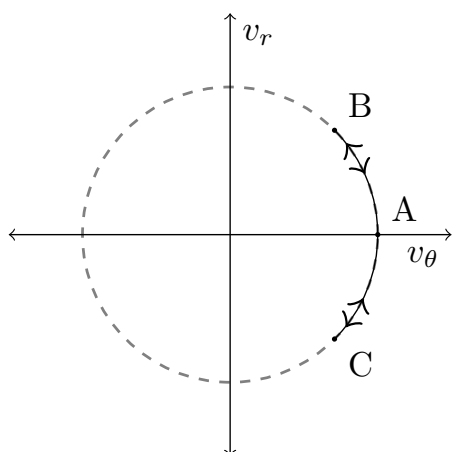

Figure 6: Engagement trajectory 2 in $\left(v_{\theta}, v_{r}\right)$ space

The rate of change of $\phi$ with respect to time varies depending on the sign of $K$. Considering the two cases separately, we have:

Case 1: $K>0$

The above equation indicates that for $K>0$ we get $\dot{\phi}>0$. This corresponds to clockwise movement in the $\left(v_{\theta}, v_{r}\right)$ plane as shown in Fig. 5 . Now, $v_{r}>0$ in the path $C-B-A$ which means instantaneous $r$ increases in this path upto point $A$ and thereafter it decreases as $v_{r}<0$ in the path $A-D-C$. Hence, $R_{\text {max }}$ occurs at $A\left(\phi=\frac{3 \pi}{2}\right)$ and $R_{\text {min }}$ occurs at $\mathrm{C}\left(\phi=\frac{\pi}{2}\right)$.

Case 2: $K \leqslant 0$

$v_{r}$ and $v_{\theta}$ are continuous functions of $\phi$. By re-arranging Eqn. (12), we get $\sin \phi=\frac{k\left(K-r^{3}\right)}{3 v r}$. Hence, from Eqn. (7) for all values of $K \leqslant 0, v_{\theta} \geqslant 0$. This indicates that the engagement geometry can exist only in the first and fourth quadrants in the $\left(v_{\theta}, v_{r}\right)$ plane. So, the only feasible inflexion point is $\phi=\frac{3 \pi}{2}$. Hence, considering the continuity of the $v_{\theta}$ and $v_{r}$ functions, we conclude that the $\left(v_{\theta}, v_{r}\right)$ plot oscillates about $\phi=\frac{3 \pi}{2}$ as shown in Fig. 6. Now, $v_{r}>0$ in the path $A-B-A$ which means instantaneous $r$ increases in this path till the second time it reaches point $A$. Thereafter, it decreases as $v_{r}<0$ in the path $A-C-A$ till it comes to the point A for the second time. Hence, in this case, both $R_{\max }$ and $R_{\min }$ occur at $A\left(\phi=\frac{3 \pi}{2}\right)$.

Thus, by using the coverage control algorithm given in Eqn. (5), we get two types of engagement trajectories in $\left(v_{r}, v_{\theta}\right)$ plane, depending on $K$ or the initial conditions $\left(r_{0}, \phi_{0}\right)$. By differentiating Eqns. (6) and (7), we get:

$$
\begin{array}{r}
\dot{v}_{r}=-v_{\theta} \dot{\phi} \\
\dot{v}_{\theta}=v_{r} \dot{\phi}
\end{array}
$$

At the points B and C shown in Fig. $6, \dot{v}_{r}$ and $\dot{v}_{\theta}$ become zero simultaneously, which implies $\dot{\phi}=0$. Using this condition in Eqn. (13), we get the following result for the instantaneous value of $r$ :

$$
r=\left(\frac{K^{\prime}}{2}\right)^{\frac{1}{3}}
$$

where $K^{\prime}=(-K)$. Substituting eqn. (16) in eqn. (12) and further solving, we get an expression of instantaneous $\phi$ as,

$$
\sin \phi=\frac{-k}{v}\left(\frac{K^{\prime 2}}{4}\right)^{\frac{1}{3}}
$$

Eqn. (17) gives a bound on $K^{\prime}=(-K)$ as sine function can take values between -1 to 1 . Hence, the minimum value of $K$ can be obtained by setting $\sin \phi=-1$, which gives $K=-K^{\prime}=-2 \sqrt{\frac{v^{3}}{k^{3}}}$. Let us denote 
the lower value of $K$ by: $-K_{b r}=-2 \sqrt{\frac{v^{3}}{k^{3}}}$. Thus, coverage becomes feasible for negative values of $K$ only if $-K_{b r} \leqslant K \leqslant 0$. Next we address two problems: If we are given an initial condition, what will be the coverage area and if we are given an area to be covered, what should be the initial conditions to achieve the coverage?

\section{III.A. Achieving coverage for a specified $r_{0}$ and $\phi_{0}$}

Given an initial condition $\left(r_{0}, \phi_{0}\right)$, we have the following result:

Theorem 1: Given a set of initial conditions $\left(r_{0}, \phi_{0}\right)$ for an agent with kinematics (1)-(3) and a control law given by (5), coverage will be achieved for a region given by the maximum radius $R_{\max }$ and minimum radius $R_{\min }$ which are given as:

$$
\begin{aligned}
R_{\text {max }} & =a z_{1}+b z_{2} \\
R_{\text {min }} & =a z_{3}+b z_{4}
\end{aligned}
$$

where

$$
\begin{aligned}
& a=\left\{\begin{array}{ll}
\frac{-1+i \sqrt{3}}{2}, & \text { if }-K_{b r} \leqslant K \leqslant 0 \\
1, & \text { if } 0<K<\infty
\end{array} \quad z_{1}=-\left(-\frac{K}{2}+\sqrt{\frac{K^{2}}{4}-\frac{v^{3}}{k^{3}}}\right)^{\frac{1}{3}}\right. \\
& b=\left\{\begin{array}{ll}
\frac{-1-i \sqrt{3}}{2}, & \text { if }-K_{b r} \leqslant K \leqslant 0 \\
1, & \text { if } 0<K<\infty
\end{array} \quad z_{2}=-\left(-\frac{K}{2}-\sqrt{\frac{K^{2}}{4}-\frac{v^{3}}{k^{3}}}\right)^{\frac{1}{3}}\right. \\
& z_{3}= \begin{cases}z_{2} & \text { if }-K_{b r} \leqslant K \leqslant 0 \\
-\left(-\frac{K}{2}+\sqrt{\frac{K^{2}}{4}+\frac{v^{3}}{k^{3}}}\right)^{\frac{1}{3}} & \text { if } 0<K<\infty\end{cases} \\
& z_{4}= \begin{cases}z_{1} & \text { if }-K_{b r} \leqslant K \leqslant 0 \\
-\left(-\frac{K}{2}-\sqrt{\frac{K^{2}}{4}+\frac{v^{3}}{k^{3}}}\right)^{\frac{1}{3}} & \text { if } 0<K<\infty\end{cases}
\end{aligned}
$$

Proof: The calculation of $R_{\max }$ and $R_{\min }$ are done on the basis of the $\left(v_{\theta}, v_{r}\right)$ plots. Given the Figs. 5 and 6 , we have derived that $R_{\min }$ and $R_{\max }$ occur at $\phi=\pi / 2$ and $\phi=3 \pi / 2$ while $K \in(-\infty, \infty)$. Hence, we have the four cases:

$$
\begin{array}{llll}
\text { Case } & 1: & K>0, & \phi=3 \pi / 2 \\
\text { Case } & 2: & K>0, & \phi=\pi / 2 \\
\text { Case } & 3: & K \leqslant 0, & \phi=3 \pi / 2 \\
\text { Case } & 4: & K \leqslant 0, & \phi=\pi / 2
\end{array}
$$

Case 1: $\phi=3 \pi / 2$ and $K>0$

The $\left(v_{\theta}, v_{r}\right)$ plot of Figure 5 shows that instantaneous $r$ attains a maximum for $\phi=3 \pi / 2$. Substituting the value of $\phi$ in Eqn. (12), we get a cubic equation in $r$. The nature roots of Eqn. (12) can be obtained using root locus plot. In order to do that we re-arranged Eqn. (12) to get the Evans form as $\left(1+\frac{-K}{r^{3}-\frac{3 v r}{k}}=0\right)$. Hence, we get the root locus plot shown Fig. 7. It shows that for $0<K<\infty$, the equation has one positive solution. From $\left(v_{\theta}, v_{r}\right)$ plot we find that this root corresponds to the maximum value of instantaneous $r$. Solving Eqn. (12), we get the value of the root as: 


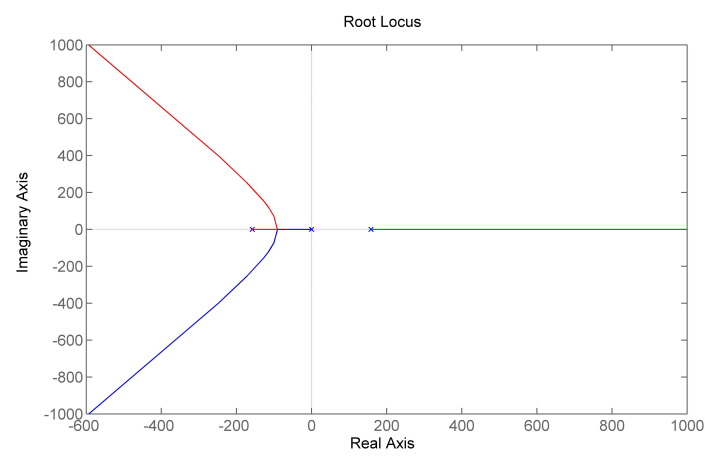

Figure 7: Root Locus for positive $\mathrm{K}$ and $\phi=3 \pi / 2$

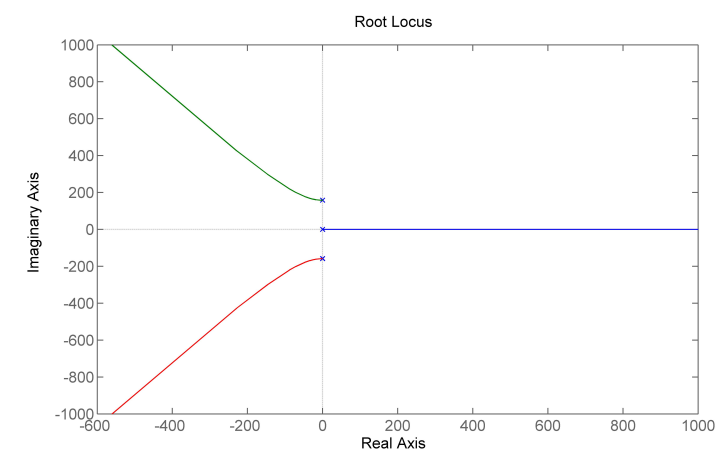

Figure 8: Root Locus for positive $\mathrm{K}$ and $\phi=\pi / 2$

$$
R_{\max }=-\left(-\frac{K}{2}+\sqrt{\frac{K^{2}}{4}-\frac{v^{3}}{k^{3}}}\right)^{\frac{1}{3}}-\left(-\frac{K}{2}-\sqrt{\frac{K^{2}}{4}-\frac{v^{3}}{k^{3}}}\right)^{\frac{1}{3}}
$$

Case 2: $\phi=\pi / 2$ and $K>0$

Proceeding the same way as for the previous case, we conclude that for $K>0, \phi=\frac{\pi}{2}$ corresponds to the minimum value of the instantaneous $r$. Hence, by substituting this condition in Eqn. (12), we get a cubic equation in $r$. To analyse the behaviour of the roots, we re-arrange Eqn. (12), to get the Evan's form as $\left(1+\frac{-K}{r^{3}+\frac{3 v r}{k}}=0\right)$. The root locus plot of the equation is given in Fig. 8. It shows that for $0<K<\infty$, the equation has one positive solution which corresponds to the minimum value of instantaneous $r$. Solving Eqn. (12), we get the value of the root as:

$$
R_{\text {min }}=-\left(-\frac{K}{2}+\sqrt{\frac{K^{2}}{4}+\frac{v^{3}}{k^{3}}}\right)^{\frac{1}{3}}-\left(-\frac{K}{2}-\sqrt{\frac{K^{2}}{4}+\frac{v^{3}}{k^{3}}}\right)^{\frac{1}{3}}
$$

Case 3: $\phi=3 \pi / 2$ and $K \leqslant 0$

The $\left(v_{\theta}, v_{r}\right)$ plot of Fig. (6) shows that for $\phi=3 \pi / 2$, the instantaneous $r$ attains both its maximum and minimum values in an alternate manner. Substituting the value of $\phi$ in Eqn. (12), we get the cubic equation in terms of $r$. To generate the root locus, we re-arranged Eqn. (12) to get the Evans form as $\left(1+\frac{K^{\prime}}{r^{3}-\frac{3 v r}{k}}=0\right)$, where $K^{\prime}=-K$. The root locus plot, shown in Fig. 9, shows that for $0 \leqslant K^{\prime} \leqslant K_{b r}$, the equation yields two positive roots indicating $R_{\max }$ and $R_{\min }$. But, beyond $K^{\prime}=K_{b r}$ which corresponds to the breakaway value of $K^{\prime}$, the two positive roots become imaginary. Hence, no value of $K^{\prime}$ greater than $K_{b r}$ gives a feasible solution. At the breakaway value of $K^{\prime}=K_{b r}=2\left(\frac{v}{k}\right)^{\frac{3}{2}}, R_{\max }$ and $R_{\min }$ become equal. Now, before solving the eqn. (12), we define two terms $z_{1}=-\left(-\frac{K}{2}+\sqrt{\frac{K^{2}}{4}-\frac{v^{3}}{k^{3}}}\right)^{\frac{1}{3}}$ and $z_{2}=-\left(-\frac{K}{2}-\sqrt{\frac{K^{2}}{4}-\frac{v^{3}}{k^{3}}}\right)^{\frac{1}{3}}$. The instantaneous value of $r$ corresponding to its maximum and minimum values are given by,

$$
\begin{aligned}
& R_{\text {max }}=\left(\frac{-1+i \sqrt{3}}{2}\right) z_{1}+\left(\frac{-1-i \sqrt{3}}{2}\right) z_{2} \\
& R_{\text {min }}=\left(\frac{-1-i \sqrt{3}}{2}\right) z_{1}+\left(\frac{-1+i \sqrt{3}}{2}\right) z_{2}
\end{aligned}
$$




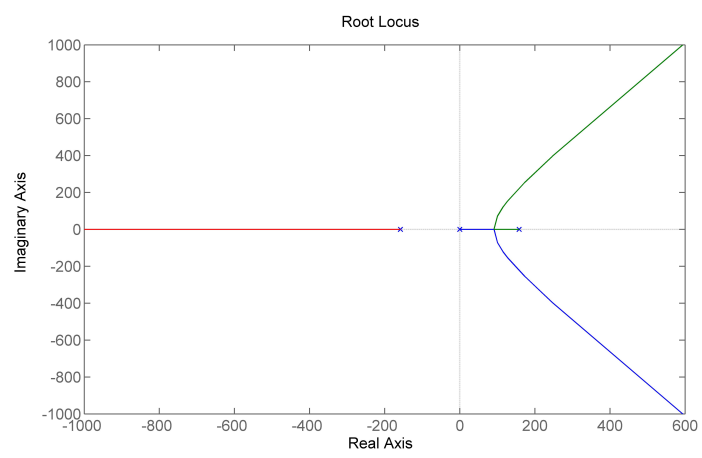

Figure 9: Root Locus for negative $\mathrm{K}$ and $\phi=3 \pi / 2$

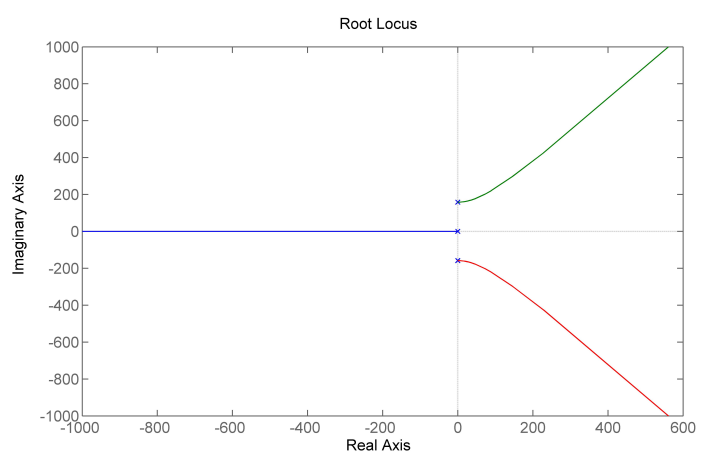

Figure 10: Root Locus for negative $\mathrm{K}$ and $\phi=\pi / 2$

Case 4: $\phi=\pi / 2$ and $K \leqslant 0$

We substituted these values in Eqn. (12) to get the Evans form as $\left(1+\frac{K^{\prime}}{r^{3}+\frac{3 v r}{k}}=0\right)$. By using the Evans form, we obtained the root locus plot as shown in Fig. 10. It shows that, no feasible roots exist. Hence, coverage cannot be attained for this configuration.

Hence, we can conclude from the four cases that given an initial condition, the maximum and minimum radii of the annular region that can be covered by the agent is as given in Eqns. (20), (21), (22) and (23).

\section{III.B. Achieving coverage with desired $R_{\max }$ and $R_{\min }$}

In the previous section, we saw that for any initial condition, we can calculate the value of $K$. Using that $K$ value, we can obtain the maximum and minimum value that the instantaneous $r$ can take. Now, if the user specifies the $R_{\max }$ and $R_{\min }$ values, it is possible to generate the initial conditions a priori by performing some calculations. But, as for the algorithm, $R_{\max }$ and $R_{\min }$ are always dependent on each other. So, once $R_{\max }$ is specified by the user, then, $R_{\min }$ can take only specific values. The relationship existing between $R_{\max }$ and $R_{\min }$ can be established as follows: For any $K$ satisfying $-K_{b r} \leqslant K \leqslant 0, \phi=\frac{3 \pi}{2}$ gives both $R_{\max }$ and $R_{\min }$. So, replacing $r$ by $R_{\max }$ or $R_{\min }$ and the corresponding value of $\phi=\frac{3 \pi}{2}$ in Eqn. (12) gives, $K=R_{\text {min }}^{3}-\frac{3 v R_{\min }}{k}=R_{\text {max }}^{3}-\frac{3 v R_{\max }}{k}$. For any value of $K$ such that $0<K<\infty$, by performing similar calculations, we get, $K=R_{\min }^{3}+\frac{3 v R_{\min }}{k}=R_{\max }^{3}-\frac{3 v R_{\max }}{k}$. Hence, given desired $R_{\max }$ or $R_{\min }$, then the parameter $K$ can be evaluated. Eqn. (11) can be re-arranged to get,

$$
\sin \phi_{0}=\frac{k\left(K-r_{0}^{3}\right)}{3 v r_{0}}
$$

Case 1: $0<K<\infty$

From the $\left(v_{\theta}, v_{r}\right)$, shown in Figure 5, we know that for $0<K<\infty,-v<v_{\theta}<v$ which implies $-1<-\sin \phi_{0}<1$ and $\phi_{0} \in(0,2 \pi)$. Hence, by using (24), we get the range of feasible values of $r_{0}$ given by,

$$
-1<\frac{k\left(K-r_{0}^{3}\right)}{3 v r_{0}}<1
$$

Case 2: $-K_{b r} \leqslant K \leqslant 0$

For this case, we get the $\left(v_{\theta}, v_{r}\right)$ as shown in Figure 6 . This indicates $0 \leqslant v_{\theta} \leqslant 1$ which implies $-1 \leqslant$ $-\sin \phi_{0} \leqslant 0$ and $\pi \in[\pi, 2 \pi]$. So, combining this result with Eqn. (24), we get,

$$
-1 \leqslant \frac{k\left(K-r_{0}^{3}\right)}{3 v r_{0}} \leqslant 0
$$

Hence, for any $r_{0}=\overline{r_{0}}$, chosen from the range of values obtained from either Eqn. (25) or eqn. (26), the appropriate value of $\phi_{0}=\overline{\phi_{0}}$ can be calculated from Eqn.(24) i.e. $\sin \overline{\phi_{0}}=\frac{k\left(K-\overline{r_{0}}{ }^{3}\right)}{3 v \bar{r}_{0}}$. Hence, for each value 


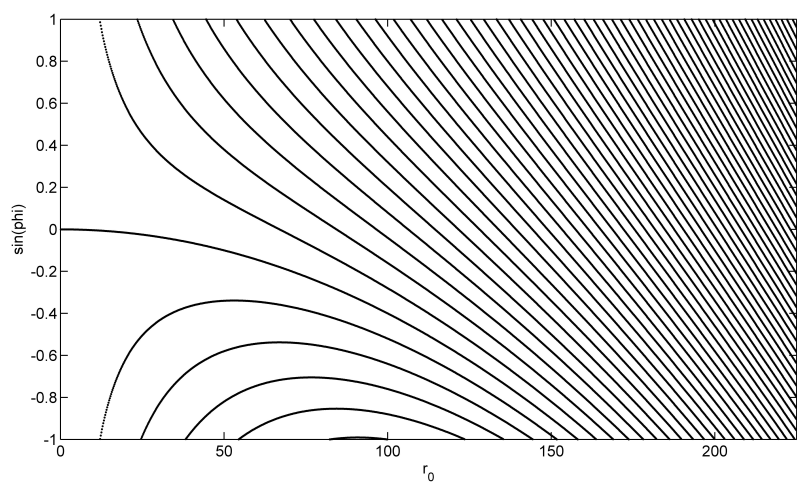

Figure 11: $\left(r_{0}, \sin \phi_{0}\right)$ for a range of $K$ values

of $K$, we get a range of feasible values of $\left(r_{0}, \sin \phi_{0}\right)$.

If we plot the feasible values of $\left(r_{0}, \sin \phi_{0}\right)$ for a range of values of $K$, we get the plot shown in Fig. 11. For $K \leqslant 0$, a particular $\sin \phi_{0}$ value can map to two values of $r_{0}$. For $K \geqslant 0$, a particular $\sin \phi_{0}$ value can map to one value of $r_{0}$. For every point in the $\left(r_{0}, \sin \phi_{0}\right)$ space with $r_{0}>0$, we get a feasible value of $K$. Hence, coverage is ensured for every feasible point in the $\left(r_{0}, \sin \phi_{0}\right)$ space. The method illustrated in this section can be summarised by the algorithm which follows: Given $R_{\max }$, then the initial conditions necessary to achieve coverage of the region of interest are given by:

1. $K=R_{\max }^{3}-\frac{3 v R_{\max }}{k}$

2. If $K>0$, then $R_{\text {min }}^{3}+\frac{3 v R_{\min }}{k}-K=0$, else $R_{\text {min }}^{3}-\frac{3 v R_{\min }}{k}-K=0$. Solution of the appropriate equation gives $R_{\text {min }}$.

3. If $K>0$, then choose any $r_{0}$ such that $-1<\frac{k\left(K-r_{0}^{3}\right)}{3 v r_{0}}<1$, else choose $r_{0}$ such that $-1 \leq \frac{k\left(K-r_{0}^{3}\right)}{3 v r_{0}} \leq 0$.

4. The value of $\phi_{0}$ is given by, $\phi_{0}=\arcsin \left(\frac{k\left(K-r_{0}{ }^{3}\right)}{3 v r_{0}}\right)$.

Given $R_{\text {min }}$, then the initial conditions necessary to achieve coverage of the region of interest are given by:

1. $K=R_{\text {min }}^{3}-\frac{3 v R_{\max }}{k}$ or $K=R_{\min }^{3}+\frac{3 v R_{\max }}{k}$.

2. Then $R_{\max }^{3}-\frac{3 v R_{\max }}{k}-K=0$. Solution of the equation gives $R_{\max }$.

3. If $K>0$, then choose any $r_{0}$ such that $-1<\frac{k\left(K-r_{0}^{3}\right)}{3 v r_{0}}<1$, else choose $r_{0}$ such that $-1 \leq \frac{k\left(K-r_{0}^{3}\right)}{3 v r_{0}} \leq 0$.

4. The value of $\phi_{0}$ is given by, $\phi_{0}=\arcsin \left(\frac{k\left(K-r_{0}{ }^{3}\right)}{3 v r_{0}}\right)$.

Once $r_{0}$ is obtained, any starting point, $\left(x_{0}, y_{0}\right)$, in the cartesian coordinate system can be chosen such that $r_{0}^{2}=x_{0}^{2}+y_{0}^{2}$. Then, $\theta_{0}$ is calculated by $\theta=\arctan \left(\frac{y_{0}}{x_{0}}\right)$. Now, the $\phi_{0}$, obtained using the algorithm, is used to generate the heading angle of the agent given by, $\alpha_{0}=\left(\theta_{0}+\phi_{0}\right)$. Thus, the initial coordinates obtained are given by: $\left(x_{0}, y_{0}, \alpha_{0}\right)$.

\section{III.C. Critical Initial Position}

Critical Initial Position refers to that value of $\left(r_{0}, \phi_{0}\right)$ for which $R_{\max }$ and $R_{\min }$ are equal. This makes the agent move on a circular path around the stationary target. Also, it is interesting to note that for these initial coordinates, we get $r_{0}=R_{\max }=R_{\min }$.

Theorem 2: For $r_{0}=\sqrt{\frac{v}{k}}$ and $\phi_{0}=3 \pi / 2$, the trajectory of an agent with kinematics (1)-(3) and a control law given by (5), is a circle of radius $\sqrt{\frac{v}{k}}$.

Proof: Given the initial conditions $r_{0}=\sqrt{\frac{v}{k}}$ and $\phi_{0}=\frac{3 \pi}{2}$. From (11), we get $K=-2 \sqrt{\frac{v^{3}}{k^{3}}}$. In order 
to calculate the $R_{\max }$ and $R_{\min }$ using the $K$ and $\phi_{0}$ values, we refer to Theorem 1 . Since $K \in\left[-K_{b r}, 0\right]$, from case III of theorem, we get that $K=-2 \sqrt{\frac{v^{3}}{k^{3}}}$ corresponds to the breakaway point of the root locus given in Fig. 9. At the breakaway point, two roots of the Eqn. (12) are real and equal and the third root is imaginary, as shown in Fig. 9. Hence, by using Eqns. (22) and (23) for the given initial conditions, we get $R_{\max }$ and $R_{\min }$ as:

$$
R_{\max }=R_{\min }=\sqrt{\frac{v}{k}}
$$

The given initial conditions, which make the agent circle around the stationary target, are referred to as the critical initial position, given by,

$$
\begin{aligned}
& r_{0}=r_{c r}=\sqrt{\frac{v}{k}} \\
& \sin \phi_{0}=-1 \Rightarrow \phi_{0}=3 \pi / 2
\end{aligned}
$$

From (27) and (28), we conclude that $r_{0}=R_{\max }=R_{\min }$. Hence, the autonomous agent circles around the target with a fixed radius of $\sqrt{\frac{v}{k}}$ for all time $t>0$.

\section{Special case: Behaviour of the system when $\phi_{0}=3 \pi / 2$}

The coverage algorithm, designed here, ensures coverage for all feasible values of initial radial distance, $r_{0}$ and $\phi_{0}$. But, the particular situation of $\phi_{0}=3 \pi / 2$ gives interesting results for $R_{\max }$ and $R_{\min }$. Also, in the previous section, we saw that for $\phi_{0}=3 \pi / 2$ the agent revolves around the target with a radius $r=\sqrt{\frac{v}{k}}$. Now, we have $K=\left(r_{0}^{3}-\frac{3 v r_{0}}{k}\right)$. Hence, for $0 \leqslant r_{0} \leqslant \sqrt{\frac{3 v}{k}}$, we get $K \leqslant 0$ and for $r_{0}>\sqrt{\frac{3 v}{k}}$, we get $K>0$.

\section{IV.A. Maximum \& Minimum radii for any initial radial distance, $r_{0}$}

In this section, we have derived the $R_{\max }$ and $R_{\min }$ for $\phi_{0}=\frac{3 \pi}{2}$. The $R_{\max }$ and $R_{\min }$ follow patterns as we vary the initial radial distance $r_{0}$.

Case 1: $K \leqslant 0$

From the $\left(v_{\theta}, v_{r}\right)$ plot of Fig. 6 , we get $\phi=\frac{3 \pi}{2}$ corresponds to both $R_{\max }$ and $R_{\min }$. Simplifying the eqns. (22) and (23) yields two positive roots, $R_{\max }$ and $R_{\min }$ :

$$
r_{2}=\frac{-r_{1}=r_{0}}{2}
$$

Now, depending on $r_{0}$, one of the roots becomes $R_{\max }$ and the other $R_{\min }$. For initial radial distance less than $r_{0}=r_{c r}, r=r_{0}$ becomes $R_{\min }$ and the other root becomes $R_{\max }$ which exactly reverses for $r_{c r} \leq r_{0} \leq \sqrt{\frac{3 v}{k}}$. So, the behaviour of the $R_{\max }$ and $R_{\min }$ vary in accordance to the initial radial distance from the target.

Case 2: $\quad K>0$

The $\left(v_{\theta}, v_{r}\right)$ plot of Figure 5 gives $\phi=\frac{3 \pi}{2}$ indicates $R_{\max }$ and $\phi=\frac{\pi}{2}$ indicates $R_{\min }$. Simplifying the Eqns. (20) and (21), we get the two positive roots as:

$$
\begin{array}{r}
R_{\max }=r_{0} \\
R_{\min }=-\left(\frac{K}{2}+\sqrt{\frac{K^{2}}{4}+\frac{v^{3}}{k^{3}}}\right)^{\frac{1}{3}}-\left(\frac{K}{2}-\sqrt{\frac{K^{2}}{4}+\frac{v^{3}}{k^{3}}}\right)^{\frac{1}{3}}
\end{array}
$$

All the above results were verified from their second derivatives. 


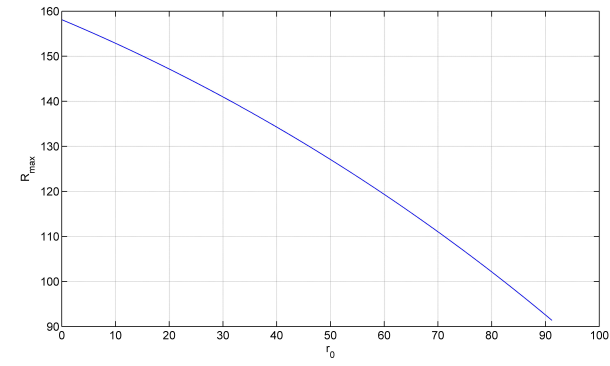

Figure 12: $R_{\max }$ for $\phi=3 \pi / 2,0<r_{0}<\sqrt{\frac{v}{k}}$

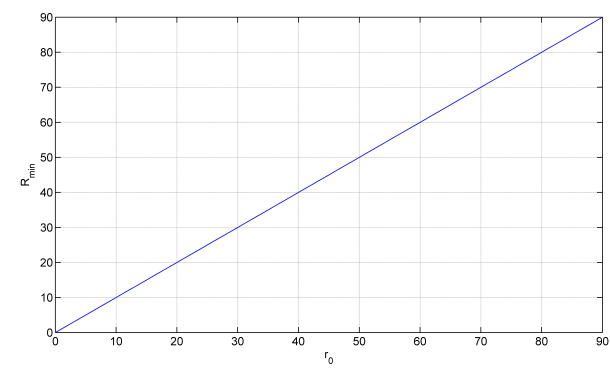

Figure 14: $R_{\text {min }}$ for $\phi=3 \pi / 2$ and $0<r_{0}<\sqrt{\frac{v}{k}}$

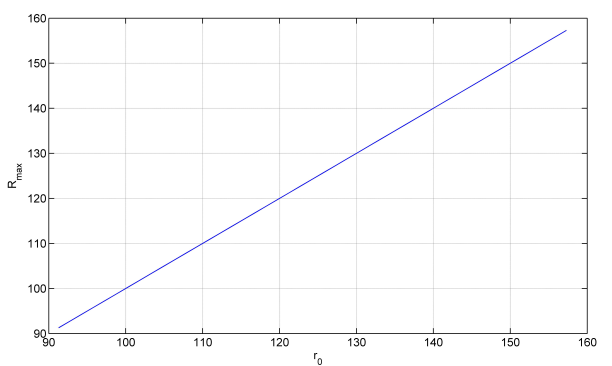

Figure 13: $R_{\max }$ for $\phi=3 \pi / 2, \sqrt{\frac{v}{k}}<r_{0}<\sqrt{\frac{3 v}{k}}$

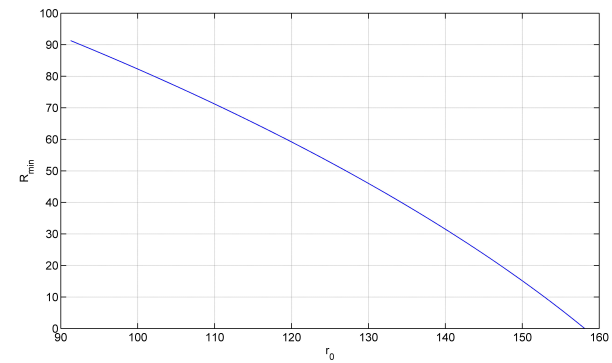

Figure 15: $R_{\min }$ for $\phi=3 \pi / 2$ and $\sqrt{\frac{v}{k}}<r_{0}<\sqrt{\frac{3 v}{k}}$

\section{IV.B. Agent restricts itself within a stretch of radius $\sqrt{\frac{3 v}{k}}$}

Given, $\phi_{0}=\frac{3 \pi}{2}$, the trajectory of the agent has a peculiar behaviour: For $0 \leqslant r_{0} \leqslant \sqrt{\frac{3 v}{k}}$, the maximum radial distance that can be covered by the agent can reach a maximum of $R_{\max }=\sqrt{\frac{3 v}{k}}$ and never exceed it. In a similar manner, the minimum radius that the agent can reach is $R_{\min }=0$.

Corollary 1: Given $\phi_{0}=\frac{3 \pi}{2}$, for any initial position $r_{0} \in\left[0, \sqrt{\frac{3 v}{k}}\right]$, the trajectory of the agent is bound by a circle of radius $\sqrt{\frac{3 v}{k}}$.

Proof: In the previous section, we derived the values of $R_{\max }$ and $R_{\min }$ for any arbitrary initial position $r_{0}$. Eqns. (29) and (30) establish a relationship of $R_{\max }$ and $R_{\min }$ with $r_{0}$. The nature of the variation $R_{\max }$ and $R_{\min }$ with respect to $r_{0}$ can be explained by plotting (29) and (30). Fig. 12 shows the variation of $R_{\max }$ for $r_{0} \in\left[0, \sqrt{\frac{v}{k}}\right]$ and Fig. 13 shows the same for $r_{0} \in\left[\sqrt{\frac{v}{k}}, \sqrt{\frac{3 v}{k}}\right]$.

For $r_{0} \in\left[0, \sqrt{\frac{v}{k}}\right]$, Fig. 14 shows variation of $R_{\min }$ with respect to $r_{0}$ and Fig. 15 shows variation of $R_{\min }$ for $r_{0} \in\left[\sqrt{\frac{v}{k}}, \sqrt{\frac{3 v}{k}}\right]$. From the plots, it is clear that $\max \left(R_{\max }=\sqrt{\frac{3 v}{k}}\right)$ and $\min \left(R_{\min }\right)=0$.This ensures that if the agent starts with $\phi_{0}=3 \pi / 2$, then for any value of $r_{0}$, the agent will always remain within a circle of radius $r=\sqrt{\frac{3 v}{k}}$.

For the particular case of $r_{0}=0$ and $\phi_{0}=\frac{3 \pi}{2}$, since $r_{0} \in\left[0, \sqrt{\frac{v}{k}}\right]$ we refer to Eqn. (29), to get $R_{\max }=\sqrt{\frac{3 v}{k}}$ and $R_{\text {min }}=0$. This makes the agent cover the entire area of the circle of radius $\sqrt{\frac{3 v}{k}}$ centred around the stationary target.

\section{Simulation Results}

In this section, we validate the results obtained in sections III and IV through MATLAB simulations. The simulations were performed with the parameters $h=0.01, \rho=0.3$ and $v=5$. The control algorithm developed in Eqn. (5) leads to formation of patterns around the target ensuring coverage of the area 


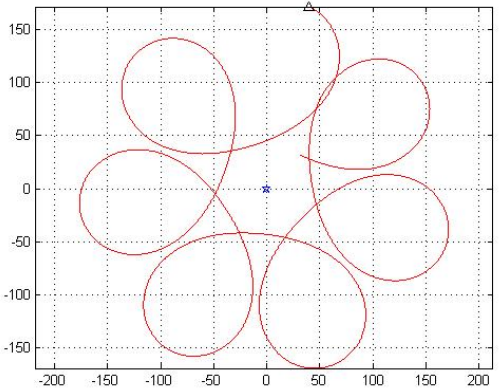

Figure 16: Trajectory plot: $r_{0}=45, \phi_{0}=\frac{110 \pi}{180}$

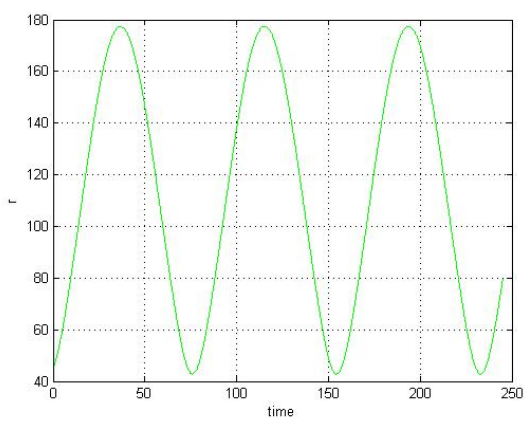

Figure 17: $r$ versus $t: r_{0}=45, \phi_{0}=\frac{110 \pi}{180}$

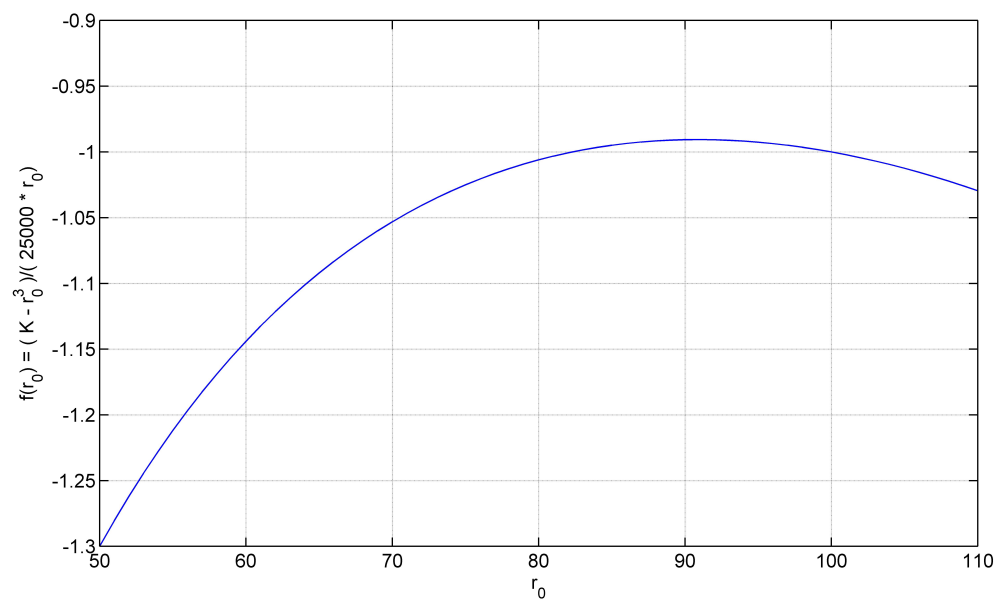

Figure 18: Range of $r_{0}$

surrounding the target.

\section{V.A. Achieving coverage for a specified $r_{0}$ and $\phi_{0}$}

Let us consider the initial conditions of $r_{0}=45$ and $\phi_{0}=\frac{110 \pi}{180}$ which give $0<K<\infty$ and hence achieve maximum and minimum radii as per Fig. 5. Now, by using Theorem 1, the maximum and minimum radii of area covered can be calculated using Eqns. (20) and (21) and are given as:

$$
\begin{aligned}
& R_{\text {max }}=177.4053 \\
& R_{\text {min }}=42.7959
\end{aligned}
$$

The trajectory plot, for the given initial conditions, is as shown in Fig. 16. From the figure, it is verified that coverage occurs. Now, the $R_{\max }$ and $R_{\min }$ can be verified from the Fig. 17 which shows the variation of instantaneous radial distance $r$, generated using MATLAB simulation.

\section{V.B. Achieving coverage with desired $R_{\max }$ and $R_{\min }$}

Let us consider a situation given by desired $R_{\max }=100$. Using $K=R_{\max }^{3}-\frac{3 v R_{\max }}{k}$, we proceed to calculate $K=-1500000$. Since, $-K_{b r} \leqslant K \leqslant 0$, using the relationship $K=R_{\text {min }}^{3}-\frac{3 v R_{\min }}{k}$, we get $R_{\min }=82.28757$. Now, $-K_{b r} \leqslant K \leqslant 0$, hence, as given in section III.B, we get the restrictions on $\phi_{0}$. Substituting the value of $K$ in Eqn. (26) gives a range of values of $r_{0}$ as shown in Fig.18. From Fig.18, a suitable value of $r_{0}=95$ is chosen. Using Eqn. (24), we get $\phi_{0}=263.0154$ degrees. Now, in simulations, using the above initial conditions, coverage is achieved, with desired $R_{\max }=100$. Hence, the result is verified by the simulation results shown in Fig. 20 and 19. 


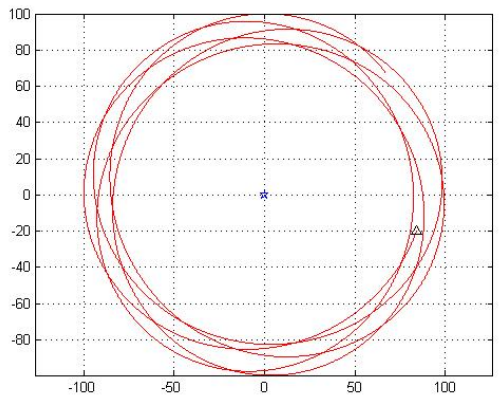

Figure 19: Trajectory plot: for $r_{0}=95, \phi_{0}=263.0154$

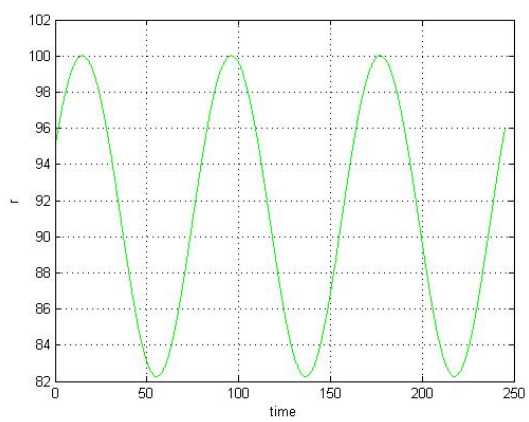

Figure 20: $r$ versus $t: r_{0}=95, \phi_{0}=263.0154$

\section{Conclusion}

In the paper, we presented a strategy that can make an autonomous agent cover the region surrounding a stationary target, using only range measurement. We derived the $R_{\max }$ and $R_{\min }$ values which characterise the region covered by the autonomous agent under the control law (5), given any initial conditions $\left(r_{0}, \phi_{0}\right)$. Similarly, we derived the initial conditions necessary to cover the region-of-interest when $R_{\max }$ and $R_{\min }$ are given. We also showed that under certain initial conditions, the agent covers the area around the target by revolving around the stationary target on a circle of fixed radius. The radius of the circular trajectory depends on the fixed parameters involved in the system of equations and, hence, can be specified by the user. As the guidance strategy uses only range measurement to achieve coverage, the energy requirements of the entire system are minimised. In the paper, the effect of initial conditions on the pattern generation has been studied. The work can be extended to study the effect of system parameters, like $\rho$, on the patterns generated. Further work can also be done to calculate the time required to cover the area specified by the user.

\section{References}

${ }^{1}$ S. C. Wong, B. A. MacDonald, "A topological coverage algorithm for mobile robots," Proceedings of the IEEW/RSJ International Conference on Intelligent Robots and Systems, Vol. 2, 1685-1690, 2003.

${ }^{2}$ A. Zelinsky, R.A. Jarvis, J. C. Byrne, S. Yuta., "Planning paths of complete coverage of an unstructured environment by a mobile robots," Proceedings of International Conference on Advanced Robotics, 533-538, 1993.

${ }^{3}$ C. Stachniss and W. Burgard, "Mapping and Exploration with Mobile Robots using Coverage Maps," Proceedings of the IEEW/RSJ International Conference on Intelligent Robots and Systems, Vol. 1, 1127-1132, 2003.

${ }^{4}$ S. Rutishauser , N. Correll and A. Martinoli, "Collaborative coverage using a swarm of networked miniature robots," Robotics and Autonomous Systems, Vol. 57, 517-525, 2009.

${ }^{5}$ P. H. Batavia, S. A. Roth, S. Singh, "Autonomous Coverage Operations In Semi-Structured Outdoor Environments," Proceedings of the IEEW/RSJ International Conference on Intelligent Robots and Systems, 743-749, 2002.

${ }^{6}$ Ercan U. Acar, Howie Choset and Prasad N. Atkar, "Complete Sensor-based Coverage with Extended-range Detectors: A Hierarchical Decomposition in Terms of Critical Points and Voronoi Diagrams," Proceedings of the IEEW/RSJ International Conference on Intelligent Robots and Systems, 1305-1311, 2001

${ }^{7}$ S. S. Dhillon and K. Chakrabarty, "Sensor Placement for Effective Coverage and Surveillance in Distributed Sensor Networks," Proceedings of IEEE Wireless Communications and Networking Conference, 1609-1614, 2003.

${ }^{8}$ Chih-Chung Lai, Chuan-Kang Ting and Ren-Song Ko, "An Effective Genetic Algorithm to Improve Wireless Sensor Network Lifetime for Large-Scale Surveillance Applications," IEEE Congress on Evolutionary Computation, 3531-3538, 2007.

${ }^{9}$ Wei Wang, Vikram Srinivasan, Bang Wang, and Kee-Chaing Chua, "Coverage for Target Localization in Wireless Sensor Networks," IEEE Transactions on Wireless Communications, Vol. 7, No. 2, 667-676, 2008.

${ }^{10}$ Ting Yan, Tian He and John A. Stankovic, "Differentiated Surveillance for Sensor Networks," First ACM Conference on Embedded Networked Sensor Systems, 51-62, 2003. 